Portland State University

PDXScholar

8-1-2010

\title{
Experimental Observations of Active Invariance Striations in a Tank Environment
}

Jorge E. Quijano

Portland State University

Richard L. Campbell

Portland State University, rlc3@pdx.edu

Tobias G. Oesterlein

Portland State University

Lisa M. Zurk

Portland State University, zurkl@pdx.edu

Follow this and additional works at: https://pdxscholar.library.pdx.edu/ece_fac

Part of the Electrical and Computer Engineering Commons

Let us know how access to this document benefits you.

Citation Details

Quijano, J. E., Campbell Jr, R. L., Oesterlein, T. G., \& Zurk, L. M. (2010). Experimental observations of active invariance striations in a tank environment. The Journal of the Acoustical Society of America, 128, 611.

This Article is brought to you for free and open access. It has been accepted for inclusion in Electrical and Computer Engineering Faculty Publications and Presentations by an authorized administrator of PDXScholar. Please contact us if we can make this document more accessible: pdxscholar@pdx.edu. 


\title{
Experimental observations of active invariance striations in a tank environment
}

\author{
Jorge E. Quijano, ${ }^{\text {a) }}$ Richard L. Campbell, Jr., Tobias G. Oesterlein, and Lisa M. Zurk \\ Department of Electrical Engineering, Northwest Electromagnetics and Acoustics Research Laboratory, \\ Portland State University, Portland, Oregon 97201-0751
}

(Received 11 September 2009; revised 4 March 2010; accepted 20 May 2010)

\begin{abstract}
The waveguide invariant in shallow water environments has been widely studied in the context of passive sonar. The invariant provides a relationship between the frequency content of a moving broadband source and the distance to the receiver, and this relationship is not strongly affected by small perturbations in environment parameters such as sound speed or bottom features. Recent experiments in shallow water suggest that a similar range-frequency structure manifested as striations in the spectrogram exists for active sonar, and this property has the potential to enhance the performance of target tracking algorithms. Nevertheless, field experiments with active sonar have not been conclusive on how the invariant is affected by the scattering kernel of the target and the sonar configuration (monostatic vs bistatic). The experimental work presented in this paper addresses those issues by showing the active invariance for known scatterers under controlled conditions of bathymetry, sound speed profile and high SNR. Quantification of the results is achieved by introducing an automatic image processing approach inspired on the Hough transform for extraction of the invariant from spectrograms. Normal mode simulations are shown to be in agreement with the experimental results. (C) 2010 Acoustical Society of America.
\end{abstract}

[DOI: $10.1121 / 1.3455813$ ]

PACS number(s): 43.30.Vh, 43.30.Bp, 43.30.Zk, 43.60.Ac [DRD] Pages: 611-618

\section{INTRODUCTION}

Striations of constant intensity are often present in the spectrograms formed from passive or active acoustic intensity in underwater waveguides. These striations are evidence of a simultaneous range-frequency constraint for the propagation of waves in the water channel, and they are the result of constructive and destructive interference between propagating modes.

For passive sonar, this effect has been described with the waveguide invariance theory introduced by Chuprov ${ }^{1}$ and popularized by Brekhovskikh, ${ }^{2}$ and some work has been done to characterize ${ }^{3-5}$ and to use this phenomena for multiple applications. ${ }^{6-8}$

A similar time-frequency structure has been found in active sonar data from recent experiments in the Malta Plateau area ${ }^{9,10}$ in shallow water. For the active invariance phenomena, a broadband pulse propagates from source-to-target, undergoes mode and azimuth dependent scattering and then propagates from target-to-receiver, preserving the timefrequency constraint along this process. Understanding of the active waveguide invariant would allow improvement of tracking techniques ${ }^{11}$ and mitigation of the effect of bottom reverberation. ${ }^{12}$ Theoretical work ${ }^{13}$ has been done to model the active sonar waveguide invariant $\gamma(\gamma$ is defined as the active invariant, similar to $\beta$ for passive scenarios), and this paper describes a series of active sonar tank experiments designed to explore the invariant in monostatic and bistatic sonar configurations.

\footnotetext{
a) Author to whom correspondence should be addressed. Electronic mail: jorgeq@pdx.edu
}

To this date, most of the research on the waveguide invariant has been done for passive sonar at low frequencies, and it is relatively easy to observe striation patterns due to low frequency "sources" such as the noise produced by moving ships. In experiments with active sonar at low and mid frequencies, however, the experimental conditions required to visualize the characteristic striation pattern in the bistatic spectrogram are more challenging. ${ }^{9}$ The width and the spacing between striations are frequency dependent: the excitation of more modes due to the use of higher frequencies (i.e., $>0.5 \mathrm{kHz}$ ) in active sonar results in thinner striations that are harder to observe. In addition, since the spectrogram is a time-frequency representation of the received intensity, there are minimum requirements for the ping rate and the frequency band that allow visual construction of the striations.

Sea experiments in shallow water have shown the existence of the active invariance phenomena. Spectrograms obtained with a monostatic configuration at low frequency ( $<700 \mathrm{~Hz}$ ) during the SWAC experiment ${ }^{9}$ exhibited constant intensity striations, and further experiments have shown the active invariance for a fully bistatic configuration at mid-frequencies. ${ }^{10}$ Active sonar tank experiments have also been conducted ${ }^{14}$ to recreate the time-frequency structure observed in reverberation time series from sea experiments. ${ }^{15}$

Despite the strong evidence of the active invariant phenomena up to this date, some features that require further attention are common among field experiments. First, the use of complicated targets with unknown scattering properties has precluded assessing the effect of the target scattering kernel on the spectrogram, which is one of the main questions regarding whether there is a true active invariant. Second, current experimental work has not validated some of the 
predictions from theoretical modeling ${ }^{13}$ of the active invariant [indicated in Section II B]. Third, the clarity of the striations in active spectrograms from sea experiments is much lower than that typically observed in passive spectrograms, leading to speculation to explain this behavior (i.e., low SNR and other factors such as range-dependent bathymetry or unintended motion of the source/receivers).

This paper attempts to address the first two points by performing laboratory experiments that show striation patterns in monostatic and bistatic spectrograms corresponding to different known targets, water depths and frequency bands. The proposed setup results in spectrograms with similar quality for both the active and passive scenarios, and therefore excludes the experimental issues raised on the third point, as well as the effect of Doppler shifting, surface/ bottom roughness, and vibration of the sensors or the target, which can be addressed in future laboratory work.

In this series of experiments, broadband pulses were reflected off moving targets of different shape, and the corresponding spectrograms exhibit a range-frequency striated structure similar to the ones observed in passive sonar lofargrams. Ultrasound frequencies of 250 and $500 \mathrm{kHz}$ were used to model sea experiments at $500 \mathrm{~Hz}$ and $1 \mathrm{kHz}$, which is a reasonable factor for scaling the depth of the water column. Some geoacoustic parameters such as the grain size of the sand or the frequency dependent attenuation at the bottom cannot be scaled in the laboratory setup, but regardless of those limitations, tank experiments have the advantage of a controlled environment that removes some of the uncertainties from field experiments.

Analysis of experimental spectrograms requires the estimation of the invariant parameter. In this work, an image processing method inspired in the Hough transform ${ }^{10,16}$ is introduced for automatic extraction. The method is based on finding the value of $\gamma$ that results in contour curves in the time-frequency axis, along which variation in intensity is a minimum.

This paper is organized as follows: Section II is a summary of the normal mode theory required to run simulations of the experiments and to relate them to the waveguide invariant. Section III describes the experimental set-up and the corresponding results are shown in section IV. Section V presents the method developed for the analysis of the spectrograms and extraction of the invariant parameter, and section VI corresponds to conclusions. Appendix summarizes the derivation of the bistatic invariant $\gamma$.

\section{NORMAL MODE THEORY FOR WAVEGUIDE INVARIANCE}

\section{A. Passive sonar}

Normal mode theory can be utilized to derive an expression for the waveguide invariant $\beta$ and to generate simulated spectrograms. The acoustic pressure at range $r$ and depth $z_{r}$ due to a point source located at range $r=0$ and depth $z_{s}$ is given by:

$$
p\left(r, z_{r} ; z_{s} ; \omega\right)=C \sum_{m=1}^{M} \Psi_{m}\left(z_{s}\right) \Psi_{m}\left(z_{r}\right) \frac{e^{i k_{m} r}}{\sqrt{k_{m} r}},
$$

where $k_{m}$ is the horizontal wavenumber for the $m$ th propagating mode, $\Psi_{m}(z)$ is the corresponding depth dependent mode function, $C=i e^{-i \pi / 4} / \rho \sqrt{8 \pi}$ is a normalizing constant, $M$ is the total number of propagating modes and the frequency dependency comes from the relation $k_{m}=\omega / v_{m}$ (with $v_{m}$ defined as the phase velocity of the $m$ th mode). Following Brekhovskikh, ${ }^{2}$ the scalar $\beta$ is defined as

$$
\beta=-\frac{r}{\omega} \frac{\partial I / \partial r}{\partial I / \partial \omega},
$$

where $I=\left|p\left(r, z_{r} ; z_{s} ; \omega\right)\right|^{2}$ is the acoustic intensity. By substitution of (1) into (2), and after some algebraic steps and approximations, ${ }^{2}$ (2) can be expressed as

$$
\beta=-\frac{d(1 / v)}{d(1 / u)}=\frac{r}{\omega} \frac{d \omega}{d r},
$$

where $u$ and $v$ are the average of the group and phase velocity of a certain group of modes. As pointed out in the literature, ${ }^{2}$ for an isovelocity waveguide in shallow water, the propagation at long ranges is dominated by modes with small grazing angle which results on $\beta \approx 1$. But for the tank experiments presented in this paper the higher order modes are not strongly attenuated as they propagate over short ranges, resulting in lower values of $\beta$.

\section{B. Active sonar}

In active sonar the normal modes expression for the bistatic pressure is given by

$$
\begin{aligned}
p\left(r_{1}, z_{t} ; r_{2}, z_{r} ; z_{s} ; \omega\right)= & C^{2} \sum_{m}^{M} \sum_{n}^{N} \Psi_{m}\left(z_{s}\right) \Psi_{m}\left(z_{t}\right) \\
& \times \frac{e^{i k_{m} r_{1}}}{\sqrt{k_{m} r_{1}}} S\left(\theta_{m}, \phi_{o} ; \theta_{n}, \phi\right) \Psi_{n}\left(z_{t}\right) \\
& \times \Psi_{n}\left(z_{r}\right) \frac{e^{i k_{n} r_{2}}}{\sqrt{k_{n} r_{2}}},
\end{aligned}
$$

where $r_{1}$ and $r_{2}$ are the ranges from source-to-target and target-to-receiver, $z_{t}$ is the depth of the target and $S\left(\theta_{m}, \phi_{o} ; \theta_{n}, \phi\right)$ represents the coupling of the incident mode $m$ and the scattered mode $n$, with grazing angles $\theta_{m}$ and $\theta_{n}$, respectively. The variables $\phi_{o}$ and $\phi$ represent the azimuth angle of the incident and the scattered modes. Equations (1) and (4) are used in this paper to compute simulated spectrograms for comparison with experimental data.

The wavenumbers $k_{m}$ and the mode functions $\Psi_{m}(z)$ were computed from the analytical solution for an isovelocity waveguide with rigid bottom. ${ }^{17}$

Following a procedure similar to the passive case in which the derivative of the intensity with respect to the bistatic range $r_{b}=r_{1}+r_{2}$ and the frequency are obtained from (4), the bistatic invariant ${ }^{13} \gamma$ is defined as (see Appendix for algebraic details):

Quijano et al.: Active invariance phenomena in a tank experiment 

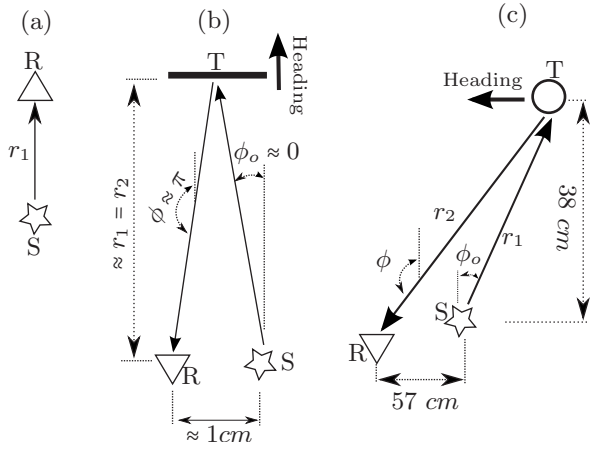

FIG. 1. Top-down diagrams of the experiments presented in this paper showing the relative position of the source $(\mathrm{s})$, target $(\mathrm{t})$ and receiver(r): (a) passive configuration; (b) active (monostatic) configuration and (c) bistatic configuration.

$$
\gamma=-\frac{r_{b}}{\omega} \frac{\partial I / \partial r_{b}}{\partial I / \partial \omega}=\beta+\epsilon,
$$

where $\epsilon$ is a function of the phase velocities, the scattering properties of the target and the mode functions. The behavior of $\epsilon$ with respect to the number of propagating modes or the scattering function of the target is a complicated relationship, but as mentioned in Appendix there are two special cases in which the value of $\epsilon$ in (5) goes to zero: for monostatic configurations $\left(r_{1}=r_{2}\right)$ and when the scattering function is given by the Kronecker function $\delta(m-n)$.

\section{EXPERIMENTAL SET-UP}

The tank experiments were conducted in a polyethylene semi-rigid tank (fresh water) with a square footprint of 3 $\times 3 \mathrm{~m}^{2}$ and a height of $30 \mathrm{~cm}$. In this work, three scenarios were considered: passive, active monostatic and bistatic experiments, illustrated in Fig. 1.

The passive experiments consisted of varying the range between the source and the receiver and at each step a linear chirp was transmitted. For the active experiments the procedure is similar, except that the receiving hydrophone and the source are fixed and the target shifts position according to the heading direction. After each movement of the target or the receiver, the tank was allowed to settle before recording the corresponding ping to limit distortion due to water motion or vibration of the sensors.

The experiments were performed at frequency bands of $200-300 \mathrm{kHz}$ and $400-600 \mathrm{kHz}$ referred in this paper as $F_{250}$ and $F_{500}$, respectively. For the $F_{250}$ a custom-made source manufactured at the Applied Physics Laboratory/University of Washington (APL/UW) was used in combination with the omni directional hydrophone B\&K8103. The experiments at the $F_{500}$ band were performed with the source TC3029 and the omni directional hydrophone TC4038 as receiver. Experimental apparatus were suspended on a frame mounted above the tank, as depicted in Fig. 2.

Both sources have some directionality, and this was used to determine $M$, the maximum number of normal modes that each source can excite in the water column, as explained next. The $F_{250}$ source radiates most of the energy within a cone of $\pm 60^{\circ}$, so it is assumed that only normal modes with a grazing angle smaller than $60^{\circ}$ (i.e., $M=19$ modes for a (a)
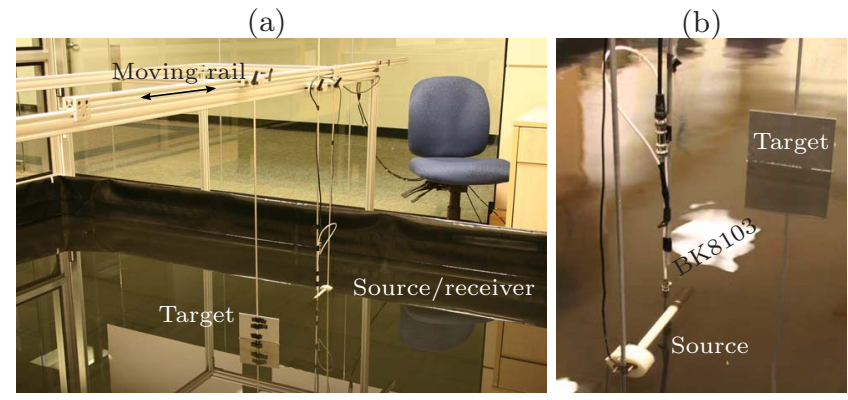

FIG. 2. (Color online) (a) Experimental setup for active tank experiments, showing the supporting aluminum frame, target and transducers; (b) Close up of the target, the BK8103 hydrophone and the source $F_{250}$ manufactured at APL/UW.

water column of $D=7.95 \mathrm{~cm}$ depth and $f=200 \mathrm{kHz}$ ) can be excited by this source. Similarly, the radiation pattern for the $F_{500}$ source concentrates around $\pm 10^{\circ}$ and for this case only $M=10$ modes are strongly excited when $D=7.95 \mathrm{~cm}$ and $f$ $=400 \mathrm{kHz}$. The value of $M$ for these and other combinations of frequency band/depth were required to run normal mode simulations of the corresponding spectrograms, by using (1) and (4).

For the active experiments, two different targets were utilized: a flat aluminum plate of $15 \times 15 \mathrm{~cm}^{2}$ and a hollow glass sphere of $8 \mathrm{~cm}$ diameter. At $f=250 \mathrm{kHz}$, those targets have a height of 25.5 and 13.6 wavelengths, respectively, which would scale to 77 and $41 \mathrm{~m}$ in a field experiment at $f=500 \mathrm{~Hz}$ (assuming $c=1500 \mathrm{~m} / \mathrm{s}$ ). The use of over sized targets responds to the goal of having scattered data with high SNR. Specific parameters and the results for the three scenarios considered in this paper are presented in the next section.

\section{EXPERIMENTAL RESULTS}

\section{A. Passive experiment for estimation of $\boldsymbol{\beta}$}

The passive experiment illustrated in Fig. 1(a) was performed to estimate the invariant corresponding to the tank setup, since $\beta$ cannot be assumed to be 1 due to the strong effect of higher order modes at small propagation ranges. The range $r_{1}$ between the source and the receiver was varied from 70 to $80 \mathrm{~cm}$ in $2 \mathrm{~mm}$ increments and then to $90 \mathrm{~cm}$ in $4 \mathrm{~mm}$ increments, and a linear chirp in the $F_{250}$ frequency band was transmitted at each step. This experiment was carried out for several water depths, all of them with similar results for the estimation of $\beta$. The results shown in this paper correspond to water depth of $D=7.95 \mathrm{~cm}$.

Figure 3(a) shows an example of the aligned matched filter output at the receiver, where the multiple arrivals observed after the direct blast are due to multiple surface/ bottom bounces. For computation of the experimental spectrogram, those multi path arrivals were time-gated using a rectangular window of $1 \mathrm{~ms}$ width, to avoid the return from the front wall of the tank (reflection from other walls arrived even later). Figure 3(b) shows the measured transmission loss (TL) at $250 \mathrm{kHz}$, obtained by Fourier analysis of windowed data, and it is compared to a normal mode simulation computed from (1) with $D=7.95 \mathrm{~cm}$. The tank environment 
(a)

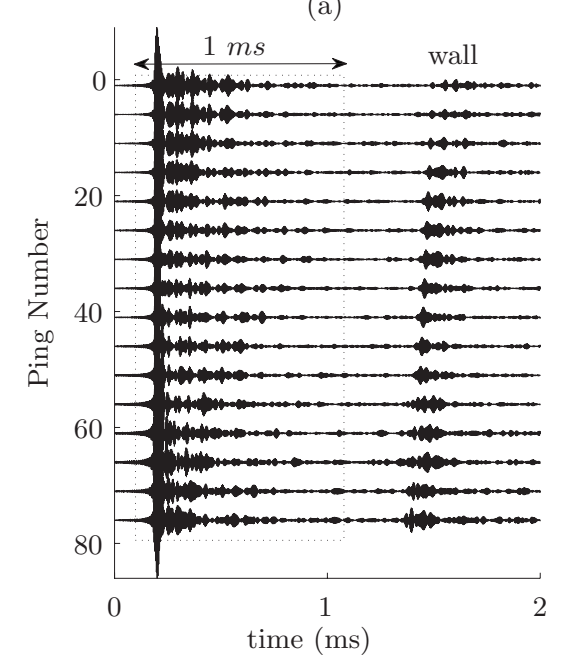

(b)

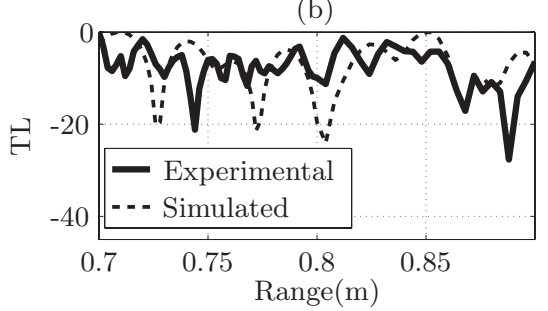

FIG. 3. (a) Pulse-compressed time series for the passive experiment, obtained by varying the range from $r_{1}=0.7 \mathrm{~m}$ (ping N.1) to $r_{1}=0.9 \mathrm{~m}$ (ping N.76). The multi-path arrivals are time gated with a rectangular window of $1 \mathrm{~ms}$ for frequency analysis. (b) Experimental transmission loss (TL) at $f$ $=250 \mathrm{kHz}$ for the windowed data, compared to a normal mode simulation.

was modeled using normal modes theory as an isovelocity channel $^{17}$ with sound speed $c=1468 \mathrm{~m} / \mathrm{s}$ and rigid bottom, and the number of modes to be included in (1) was determined based on the radiation pattern of the source as explained in Section III.

A perfect match with the experimental TL curve is hard to obtain due to factors such as changes in the water temperature or measurement mismatch of the time-varying range or the depth of the sensors. Even small perturbations in the sound speed or in the depth of the source/receiver result in considerable shifting of the location of nulls and peaks in the simulated TL curve. Despite these differences at a single frequency, the corresponding spectrograms in Fig. 4 show similar experimental/simulated striation patterns, in which the location of peaks and nulls may be different in the time/ frequency plane, but the slope of the striations is consistent, indicating that the invariant structure is robust to details of the propagation environment. Figure 4(b) will be used in Section V B to determine the value of the waveguide invariant $\beta$ for the tank setup, for comparison to the bistatic invariant $\gamma$ from the monostatic active experiments.

\section{B. Monostatic experiments}

Theoretical expressions for the active invariant show that for monostatic configurations, the value of the invariant $\gamma$ reduces to the passive invariant parameter $\beta,{ }^{13}$ and this is true also for targets with weak inter modal coupling. The results described in this section correspond to near-

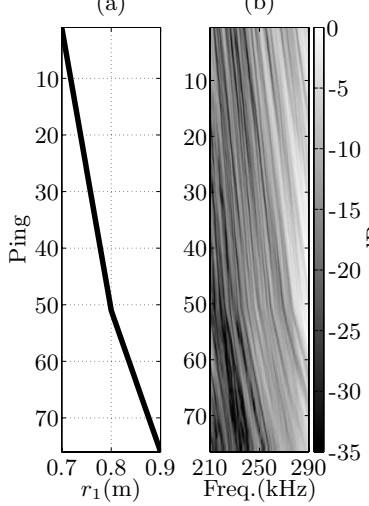

(c)

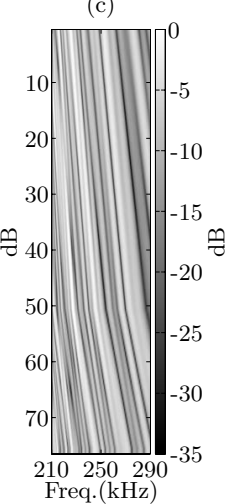

FIG. 4. Time-frequency structure for the passive experiment: (a) Sourcereceiver range vs ping number; (b) Experimental spectrogram computed from the multi path arrivals; (c) Simulated spectrogram computed from (1) and including $M=19$ modes.

monostatic configuration at the $F_{250}$ and $F_{500}$ frequency bands with $D=13.8 \mathrm{~cm}$ and $D=7.95 \mathrm{~cm}$, respectively. The purpose of the experiments is the validation of the result $\gamma$ $\approx \beta$ for monostatic configurations, and observation of the effect of exciting different number of modes in the waveguide.

The geometry corresponds to Fig. 1(b), in which the target consisted of an aluminum flat plate of dimensions 15 $\times 15 \mathrm{~cm}^{2}$ that spanned the water column from the surface to a height of $2.4 \mathrm{~cm}$ above the bottom, providing a minimum of inter modal coupling. The plate was mounted perpendicular to its axis of movement, ensuring nearly normal incidence of the acoustic pulses generated by the source. The source and the target were collocated, with a separation of less than $1 \mathrm{~cm}$. For this configuration, $\phi_{o} \sim 0^{\circ}$ and $\phi \sim 180^{\circ}$ and the plate was modeled as a perfect reflector in which no inter modal coupling takes place, so the scattering function can be written as $S\left(\theta_{m}, 180^{\circ} ; \theta_{n}, 0^{\circ}\right) \sim \delta(m-n)$.

This configuration allowed enough time for the reverberation to die out before the arrival of the target return. Similarly to the passive experiment, the pulse scattered by the target was time-gated to reject reflections from the tank walls, and (4) was utilized to compute the simulated spectrogram by assuming no inter modal coupling at the target.

Figure 5(a) shows the bistatic range $r_{b}=r_{1}+r_{2}$ and Fig.

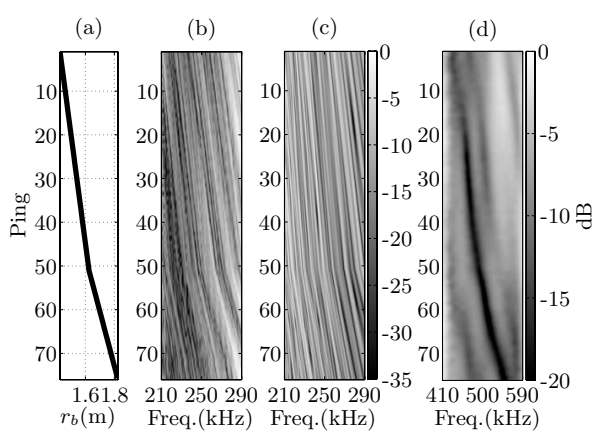

FIG. 5. Time-frequency structure corresponding to the monostatic experiments: (a) Range $r_{b}=r_{1}+r_{2}$ vs ping number; (b) Spectrogram for the monostatic experiment with $D=13.8 \mathrm{~cm}$ and $F_{250}$ frequency band; (c) Simulated spectrogram computed from (4) and including 33 modes; (d) Spectrogram for the experiment with $D=7.95 \mathrm{~cm}$ and $F_{500}$ frequency band. 


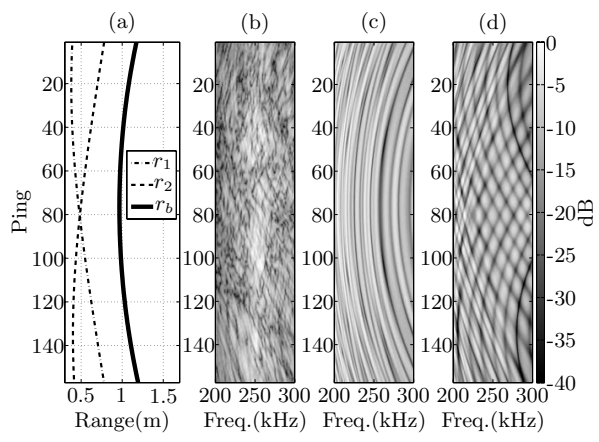

FIG. 6. Time-frequency structure corresponding to the bistatic experiment with $D=15 \mathrm{~cm}$ and $F_{250}$ band: (a) $r_{b}, r_{1}$ and $r_{2}$ vs ping number; (b) Experimental spectrogram for a spherical target; (c) Simulated spectrogram computed from (4) with $S\left(\theta_{m}, \phi_{o} ; \theta_{n}, \phi\right) \sim \delta(m-n)$; (d) Same as (c), with $S\left(\theta_{m}, \phi_{o} ; \theta_{n}, \phi\right) \sim 1$. Both simulations include 35 modes.

5(b) shows the corresponding experimental spectrogram obtained by frequency analysis of the scattered broadband pulse. Similar to field experiments, ${ }^{9,10}$ the orientation of the striations follows that of the bistatic range. Figure 5(c) shows the corresponding simulated spectrogram, computed from (4) with inputs $r_{1}, r_{2}, M=33$ modes and $S\left(\theta_{m}, \phi_{o} ; \theta_{n}, \phi\right) \sim \delta(m$ $-n)$. The slope of the striations in the simulated spectrogram match those of the experimental data.

Normally, an increase in the frequency band would result in a greater number of modes propagating in the water column. However, due to the high directionality of the $F_{500}$ source only around 10 modes are excited in the waveguide, and this results in the experimental spectrogram shown in Fig. 5(d), which has thick striations and less invariant structure as compared to the $F_{250}$ band.

\section{Bistatic experiment}

Active sonar experiments in a fully bistatic configuration were performed with the configuration in Fig. 1(c) at the $F_{250}$ frequency band using the $8 \mathrm{~cm}$ diameter glass sphere as a target. The goal of this experiment is to observe the effect of having a target with a more realistic scattering kernel (i.e., a target with finite dimensions in all axis). Figures 6(a) and 6(b) show the range as a function of ping and the corresponding spectrogram.

As predicted by theory, the resulting spectrogram depends on the geometry of the selected target and two general cases were identified: "non-mixing" targets, in which there is no inter modal coupling between incident and scattered modes, and "mixing" targets. This characteristic of the target can also be explained from (4), which can be seen as the summation of the product between the incident and the scattered pressure weighted by a factor given by the scattering function $S\left(\theta_{m}, \phi_{o}, \theta_{n}, \phi\right)$. For non-mixing targets, $S\left(\theta_{m}, \phi_{o}, \theta_{n}, \phi\right) \approx 0$ for $m \neq n$ and the factor $e^{k_{m}\left(r_{1}+r_{2}\right)}$ in (4) determines the interference pattern in the spectrogram, while for mixing targets this term becomes $e^{k_{m} r_{1}+k_{n} r_{2}}$ and is no longer a function of the bistatic range only, but the striations in the spectrogram might follow $r_{1}$ and $r_{2}$ independently.

Examples of non-mixing targets are the flat plate used for the monostatic experiments or a vertical cylinder spanning the water column: in both cases, the target is uniform along the depth axis and then incident modes are specularly reflected with respect to the vertical axis. An example of a mixing target is the glass sphere, in which an incident mode $m$ can excite scattered modes $n \neq m$.

This effect can also be observed by running normal mode simulations with $r_{1}$ and $r_{2}$ from Fig. 6(a) and computing (4) with 35 modes. The scattering function $S\left(\theta_{m}, \phi_{o} ; \theta_{n}, \phi\right) \sim \delta(m-n)$ represents a non-mixing target and the corresponding spectrogram is shown in Fig. 6(c). Notice that the striated structure follows the same orientation as the bistatic range $r_{b}$, which is clearly not the result observed in the experimental spectrogram. Simulations with a perfectly mixing scattering function like $S\left(\theta_{m}, \phi_{o} ; \theta_{n}, \phi\right) \sim 1$ (i.e., each incident mode $m$ excites all scattered modes $n$ ) result in the spectrogram shown in Fig. 6(d), in which the striations follow both $r_{1}$ and $r_{2}$, similar to the experimental result. Even though the scattering functions used in Figs. 6(c) and 6(d) do not include the azimuth dependency or the conservation of energy, they serve to illustrate the two extreme cases of how the geometry of the target might affect the spectrogram.

In the next section, a method for the estimation of the invariant is introduced and applied to the experimental spectrograms.

\section{ESTIMATION OF THE INVARIANT}

\section{A. Method for automatic estimation}

In this section, we propose a method for automatic extraction of the invariant parameter inspired in the Hough transform: ${ }^{10,16}$ the invariant is used as a parameter to define families of curves that obey the constraint in (5). Then, the spectrogram is mapped into a parameter space according to the fitness between the striations and the corresponding curves. The method proposed here uses the mean and the standard deviation of the intensity along striations to quantify this fitness.

The hypothesis to be tested is that there is some value of $\gamma$ that defines traces along striations in the spectrogram for which the intensity is nearly constant. For example, the intensity $I\left(r_{n}, z_{r} ; z_{s}, f_{n}(\gamma)\right)$ at ping $n$ is related to $I\left(r_{n-1}, z_{r} ; z_{s}, f_{n-1}(\gamma)\right)$ at the previous ping, where $f_{n-1}(\gamma)$ and $f_{n}(\gamma)$ are related by the invariant as

$$
f_{n}(\gamma)=\gamma \frac{\left[r_{n}-r_{n-1}\right]}{r_{n-1}} f_{n-1}(\gamma)+f_{n-1}(\gamma)
$$

This can be extended to more than two pings, and the standard deviation of the intensity along those traces will be minimum when the correct value of $\gamma$ is used in (6). Similarly, the mean of the intensity along those trajectories should be large. Therefore, two metrics are used to quantify the fitness of $\gamma$ to the corresponding spectrogram:

$$
\mu(f, \gamma, n)=\frac{1}{N} \sum_{j=n}^{n+N} I\left(r_{j}, f_{j}(\gamma)\right)
$$

for the mean and 
(a)

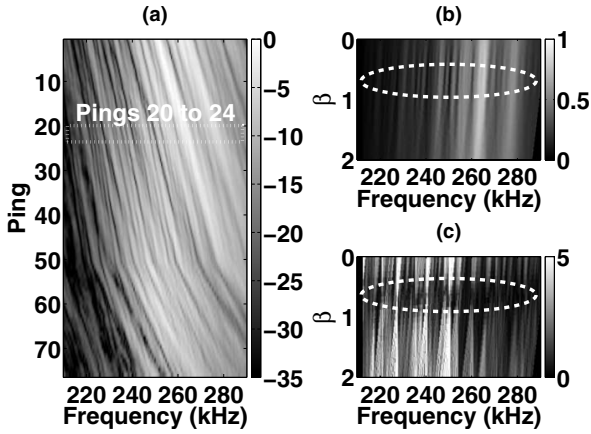

FIG. 7. (a) Experimental spectrogram from Fig. 4(b): the rectangle shows the data involved in the computation of $\mu(f, \beta, n)$ and $\sigma(f, \beta, n)$ for ping $n=20$ and $N=5$; (b) $\mu(f, \beta, n)$ evaluated at ping 20 for values of $\beta$ ranging from 0 to 2; (c) Same as (b) for $\sigma(f, \beta, n)$. The combined effect of high mean $\mu(f, \beta, n)$ and low standard deviation $\sigma(f, \beta, n)$ gives the best estimate of the parameter $\beta$.

$$
\sigma(f, \gamma, n)=\frac{1}{N} \sqrt{\sum_{j=n}^{n+N}\left[I\left(r_{j}, f_{j}(\gamma)\right)-\mu(f, \gamma, n)\right]^{2}}
$$

for the standard deviation, where $I\left(r_{j}, f_{j}(\gamma)\right)$ $\equiv I\left(r_{j}, z_{r} ; z_{s}, f_{j}(\gamma)\right)$. Note that both metrics are required, because a trajectory in a spectrogram that consists of just noise can also have small variance (i.e., the variance of the ambient noise) even in the absence of any striations, but the small mean value would allow one to recognize it as noise. In order to further reduce the variance along striations, the computations presented in this paper for $\sigma(f, \gamma, n)$ were performed with the intensity in $\mathrm{dB}$ scale (although this is not a required step).

Equations (7) and (8) were applied to the experimental spectrograms in Section IV, as exemplified in Fig. 7: Fig. 7(a) is the experimental spectrogram for the passive experiment, and Figs. 7(b) and 7(c) show the functions $\mu(f, \beta, n)$ and $\sigma(f, \beta, n)$, respectively, for ping $n=20$, with $N=5$. In this case, $\mu(f, \beta, n)$ shows local maxima at frequencies corresponding to the strongest striations in the spectrogram at $n$ $=20$ and $\beta$ around 0.65 , while $\sigma(f, \beta, n)$ shows local minima at similar frequencies and values of $\beta$.

For convenience, $\mu(f, \gamma, n)$ and $\sigma(f, \gamma, n)$ can be combined into a single parameter $L(\gamma, n)$ defined as

$$
L(\gamma, n)=\sum_{i=1}^{N_{f}}\left(\frac{\mu\left(f_{i}, \gamma, n\right)}{1+\sigma\left(f_{i}, \gamma, n\right)}\right),
$$

where $N_{f}$ is the number of frequency bins, determined by the resolution of the spectrogram in the horizontal axis, and $f_{i}$ is the corresponding frequency. In the denominator of (9), 1 is added to $\sigma\left(f_{i}, \gamma, n\right)$ to avoid division by zero in cases of small standard deviation. The function $L(\gamma, n)$ defined in (9) is the fitting parameter that considers both the mean and the standard deviation along striations, and its maximum gives an estimate of the invariant parameter for each ping $n$. In the proposed algorithm, the fitting parameter $L(\gamma, n)$ depends not only on the variance but also on the mean value of the intensity along striations. The mean value acts as a weighting factor that emphasizes the estimates corresponding to strong striations, where the SNR is higher and the potential effect of noise would be minimized.
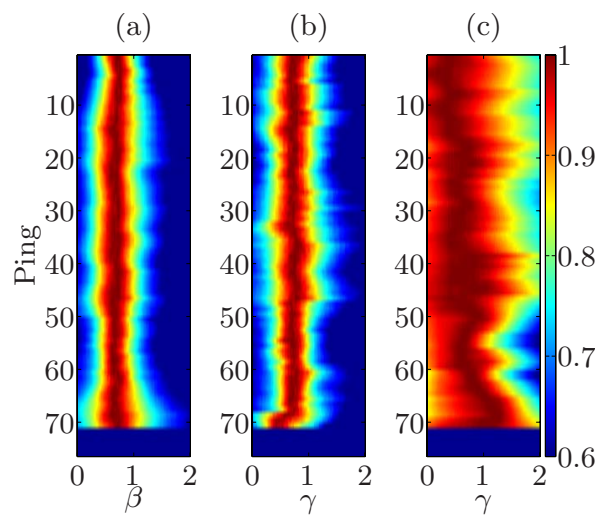

FIG. 8. (Color online) Function $L(\gamma, n)$ evaluated on the experimental spectrograms for (a) Passive experiment; (b) Monostatic experiment, $F_{250}$ and (c) Mono-static experiment, $F_{500}$. At each ping, the maximum of $L(\gamma, n)$ provides an estimate of the invariant $\beta$ (passive experiments) or $\gamma$ (monostatic experiments). The mean value and the standard deviation of the estimated invariant are summarized in Table I.

\section{B. Estimation of the invariant for passive and monostatic experiments}

Figure 8 (a) shows $L(\beta, n)$ corresponding to the passive experimental spectrogram in Fig. 4(b), while Figs. 8(b) and 8(c) show $L(\gamma, n)$ for the monostatic spectrograms in Figs. 5(b) and 5(d), respectively. Table I summarizes the mean and the standard deviation of the maximum of the estimator $L$ among all the pings. As expected, the estimates of the invariant did not vary significantly when changing from passive [Fig. 8(a)] to monostatic configuration [Fig. 8(b)] in the $F_{250}$ band. This reinforces the theoretical prediction that in a monostatic configuration $\gamma \approx \beta$.

Figure 8(c) shows the estimator $L(\gamma, n)$ for the monostatic experiment in the $F_{500}$ band. This experiment is important because due to the directionality of the source, fewer modes were excited in the water column and therefore, higher values of the invariant are expected. Due to the lack of sharp striations in the experimental spectrogram in Fig. $5(\mathrm{~d}), L(\gamma, n)$ has large variability. The sharpest striation in the experimental spectrogram goes from ping 20 to ping 76, and during those pings, the maximum of $L(\gamma, n)$ varies between 0.57 to 1.12 , confirming a shift of the invariant to higher values.

\section{Estimation of the invariant for bistatic experiments}

Estimation of $L(\gamma, n)$ was also applied to the experimental spectrogram in Fig. 6(b). Since the striation pattern shows the effect of both $r_{1}$ and $r_{2}$ as well as $r_{b}$, the fitness of $L(\gamma, n)$

TABLE I. Mean value and standard deviation of the estimated invariant, taken as the maximum of the function $L(\gamma, n)$ shown in Fig. 8.

\begin{tabular}{lccc}
\hline \hline Experiment & Invariant & Mean & Standard Dev. \\
\hline Passive & $\beta_{\text {exp }}$ & 0.69 & 0.04 \\
Monostatic, $F_{250}$ & $\gamma_{\text {exp }}$ & 0.72 & 0.06 \\
Monostatic, $F_{500}$ & $\gamma_{\text {exp }}$ & 0.63 & 0.22 \\
\hline \hline
\end{tabular}




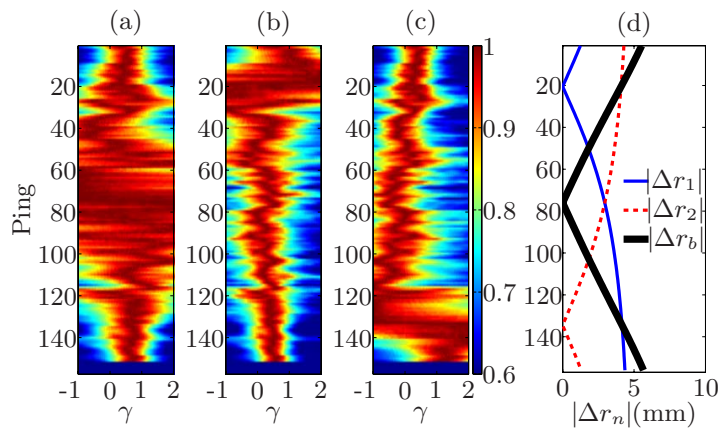

FIG. 9. (Color online) $L(\gamma, n)$ evaluated on the experimental spectrogram in Fig. 6(b): (a) $L(\gamma, n)$ when $r\left(t_{n}\right)=r_{b}\left(t_{n}\right)$ in (6); (b) $L(\gamma, n)$ when $r\left(t_{n}\right)$ $=r_{1}\left(t_{n}\right)$; (c) $L(\gamma, n)$ when $r\left(t_{n}\right)=r_{2}\left(t_{n}\right)$; (d) Rate of change of the range, defined as $\Delta r_{b}=r_{b}\left(t_{n}\right)-r_{b}\left(t_{n-1}\right), \Delta r_{1}=r_{1}\left(t_{n}\right)-r_{1}\left(t_{n-1}\right)$, and $\Delta r_{2}=r_{2}\left(t_{n}\right)$ $-r_{2}\left(t_{n-1}\right)$; the symbol $|$.$| indicates absolute value.$

to each of those ranges was quantified by substituting $r_{n}$ $=r\left(t_{n}\right)$ in (6) by $r_{b}\left(t_{n}\right), r_{1}\left(t_{n}\right)$ and $r_{2}\left(t_{n}\right)$, and the results are shown in Figs. 9(a)-9(c), respectively.

Figure $9(\mathrm{~d})$ is the absolute value of the difference $\Delta r_{n}$ $=r_{n}-r_{n-1}$, which appears in (6) and it has an effect on the variance of the estimator $L(\gamma, n)$. For example, Fig. 9(a) shows that $L(\gamma, n)$ has a large variance between pings 24 and 120 , when $\left|r_{b}\left(t_{n}\right)-r_{b}\left(t_{n-1}\right)\right|$ is small. The reason for this is that if $\Delta r_{n}$ is small in (6), then the equation losses sensitivity to the value of $\gamma$. Estimation of $\gamma$ is around 0.65 for pings 1 to 23 and 121 to 152 , corresponding to the pings where $\Delta r_{n}$ is larger.

A similar effect is observed when $L(\gamma, n)$ is fitted to $r_{1}$ and $r_{2}$ : for the case of $r_{1}$, the variance of $L(\gamma, n)$ is large between pings 1 to 60 , when the rate of change of $r_{1}$ is minimum, and the maximum of $L(\gamma, n)$ goes from $\gamma=0.4$ to $\gamma=0.6$ between pings 60 to 152 . On the other hand, when $L(\gamma, n)$ is fitted to $r_{2}$ [Fig. 9(c)], the variance is large from pings 114 to 152 , while the maximum of $L(\gamma, n)$ progressively decays from $\gamma=0.43$ to $\gamma=-0.79$ from pings 1 to 113 , indicating a better fit of the striations to $r_{2}$ during the first pings.

The results in Fig. 9 suggest an approach to identify "mixing" targets, where the $L(\gamma, n)$ fitted to each of the ranges $r_{b}, r_{1}$ and $r_{2}$ has the potential to be a signature for different kinds of scatterers. For example, computation of $L(\gamma, n)$ on the simulated spectrogram in Fig. 6(d) yields a similar result(not shown in this paper) as Fig. 9, even though the scattering function of a sphere is different than the one used to compute the simulated spectrogram.

\section{CONCLUSION}

The tank experiments presented in this paper explore the invariant phenomena under a controlled environment. Unlike the passive waveguide invariant, the active case is a more challenging scenario that includes two-way propagation of a broadband pulse, target-dependent scattering and spectrograms with thinner striations and more structure than the ones typically observed in passive sonar, due to the use of higher frequencies. The selected frequency bands with center frequency at 250 and $500 \mathrm{kHz}$ used in this paper are representative of field experiments at frequencies of $500 \mathrm{~Hz}$ and 1
$\mathrm{kHz}$, which are of current interest for active sonar applications. The active invariant in shallow water provides a simple relation for the time-frequency structure observed in spectrograms. This could be used as a robust processing technique and it has the potential to improve the performance of tracking algorithms without the requirement of precise knowledge of environmental parameters.

An important environment factor that cannot be accounted for in a tank setup is the attenuation of higher order modes at long ranges due to surface and bottom interaction, which can be relevant in field experiments. As discussed in Section II, the amount of attenuation of the higher order modes determines the effective number of modes at the receiver, and it affects the actual value of the invariant and the number and thickness of the striations in the spectrogram. This effect was observed in the tank setup by using a directional source that excites only a few modes, which resulted in spectrograms with fewer and thicker striations.

A method to quantify the invariant parameter from a spectrogram given the source-receiver range (passive experiments) or the bistatic range based on the generalized Hough transform was introduced. It was observed that the value of the fitting parameter $L(\gamma, n)$ was maximum when the timefrequency relation in (5) is satisfied. For monostatic experiments, $L(\gamma, n)$ shows that the active invariance parameter $\gamma$ approaches the well known passive parameter $\beta$.

In a bistatic scenario the scattering properties of the target strongly affect the resulting interference pattern in the spectrogram. For non-mixing targets that span the water column the resulting spectrogram follows the bistatic range as in the monostatic case. For targets with strong inter modal coupling properties, the striated structure preserves characteristics of the time-frequency structure corresponding to the trajectories source-to-target and target-to-receiver.

It is speculated that $L(\gamma, n)$ will have application in a real active sonar scenario, in which a tracking algorithm would predict multiple hypothesis (tracks) from a set of true and false detections. Given experimental spectrograms corresponding to each of the predicted tracks, false tracks could be discarded based on low values of the fitting parameter $L(\gamma, n)$.

\section{ACKNOWLEDGMENTS}

This project has been sponsored by the Office of Naval Research (ONR), Grant No. N000140510886. The authors also wish to thank Dr. Dajun Tang (APL/UW) for providing expertise and for facilitating some of the experimental equipment (200-300 kHz source and receiver BK8103.)

\section{APPENDIX: DERIVATION OF THE BISTATIC INVARIANT $\gamma$}

This appendix summarizes the derivation of the bistatic invariant $\gamma^{13}$ Following (4) and using $G_{m n}$ $\equiv S\left(\theta_{m}, \phi_{o} ; \theta_{n}, \phi\right)$, the acoustic intensity at the receiver can be written as: 


$$
I=\sum_{m, n} \sum_{p, q} B_{m n p q}\left(z_{s}, z_{t}, z_{r}\right) G_{m n} G_{p q} \cos \left(\alpha_{m n p q}\right),
$$

with

$$
\begin{aligned}
\alpha_{\text {mnpq }}=\left[\left(k_{m} r_{1}+\right.\right. & \left.\left.k_{n} r_{2}\right)-\left(k_{p} r_{1}+k_{q} r_{2}\right)\right], \\
B_{\text {mnpq }}\left(z_{s}, z_{t}, z_{r}\right)= & \zeta_{\text {mnpq }} \Psi_{m}\left(z_{s}\right) \Psi_{m}\left(z_{t}\right) \Psi_{n}\left(z_{t}\right) \\
& \times \Psi_{n}\left(z_{r}\right) \Psi_{p}^{*}\left(z_{s}\right) \Psi_{p}^{*}\left(z_{t}\right) \Psi_{q}^{*}\left(z_{t}\right) \Psi_{q}^{*}\left(z_{r}\right),
\end{aligned}
$$

$$
\zeta_{m n p q}=\frac{C^{4}}{\sqrt{k_{m} k_{n} k_{p} k_{q} r_{1}^{2} r_{2}^{2}}}
$$

where the mode term $B_{\text {mnpq }}$ is assumed to be approximately independent of frequency. Equation (A1) is similar to the intensity for passive sonar, except that it contains a double summation due to the two propagation legs of the bistatic geometry. By defining the bistatic range $r=r_{1}+r_{2}$ and difference $r_{d}=r_{1}-r_{2}, \alpha_{m n p q}$ can be written as:

$$
\begin{aligned}
\alpha_{\text {mnpq }}= & {\left[\left(k_{m}+k_{n}\right)-\left(k_{p}+k_{q}\right)\right] \frac{r}{2} } \\
& +\left[\left(k_{m}-k_{n}\right)-\left(k_{p}-k_{q}\right)\right] \frac{r_{d}}{2} .
\end{aligned}
$$

The derivatives of the intensity with respect to the bistatic range $r$ and the frequency $\omega$ are then given by

$$
\begin{aligned}
\frac{\partial I}{\partial r}= & -\sum_{m, n} \sum_{p, q} B_{m n p q}\left(z_{s}, z_{t}, z_{r}\right) G_{m n} G_{p q} \sin \left(\alpha_{m n p q}\right) \omega \\
& \times\left[\frac{1}{v_{m n}}-\frac{1}{v_{p q}}\right], \\
\frac{\partial I}{\partial \omega}= & -\sum_{m, n} \sum_{p, q} B_{m n p q}\left(z_{s}, z_{t}, z_{r}\right) G_{m n} G_{p q} \sin \left(\alpha_{m n p q}\right) r \\
& \times\left[\left(\frac{1}{u_{m n}}-\frac{1}{u_{p q}}\right)+\left(\frac{1}{u_{m q}}-\frac{1}{u_{n p}}\right) \frac{r_{d}}{r}\right],
\end{aligned}
$$

with

$$
\frac{1}{v_{i j}}=\frac{1}{2}\left(\frac{1}{v_{i}}+\frac{1}{v_{j}}\right), \quad \frac{1}{u_{i j}}=\frac{1}{2}\left(\frac{1}{u_{i}}+\frac{1}{u_{j}}\right) .
$$

Similarly to (2), the bistatic invariant $\gamma$ can be defined as

$$
\gamma=-\frac{r}{\omega} \frac{\partial I / \partial r}{\partial I / \partial \omega}=\beta+\epsilon
$$

where:

$$
\epsilon=-\beta \frac{b}{a+b},
$$

$$
\begin{aligned}
a= & \sum_{m, n} \sum_{p, q} B_{m n p q}\left(z_{s}, z_{t}, z_{r}\right) G_{m n} G_{p q} \sin \left(\alpha_{m n p q}\right) \\
& \times\left[\frac{1}{v_{m n}}-\frac{1}{v_{p q}}\right], \\
b= & \sum_{m, n} \sum_{p, q} B_{m n p q}\left(z_{s}, z_{t}, z_{r}\right) G_{m n} G_{p q} \sin \left(\alpha_{m n p q}\right) \frac{r_{d}}{r} \\
& \times\left[\frac{1}{v_{m q}}-\frac{1}{v_{n p}}\right] .
\end{aligned}
$$

From (A12), two special cases for the value of $\gamma$ are obtained: when $r_{1}=r_{2}$, the value of $\epsilon$ is zero (since $r_{d}=r_{1}$ $-r_{2}$ ), and therefore, it is expected to have $\gamma=\beta$. The second case is when the scattering function is given by the Kronecker function (no inter modal coupling), which implies that $v_{m q}=v_{n p}$ and again, $\epsilon=0$.

${ }^{1}$ S. D. Chuprov, "Interference structure of a sound field in a layered ocean," in Ocean Acoustics. Current State, edited by L. M. Brekhovskikh and I. B. Andreeva (Nauka, Moscow, 1982), pp. 71-91.

${ }^{2}$ L. M. Brekhovskikh and Y. P. Lysanov, Fundamentals of Ocean Acoustics, 3rd ed. (Springer, New York, 2002), Chap. 6, pp. 118-143.

${ }^{3}$ D. Rouseff and R. C. Spindel, "Modeling the waveguide invariant as a distribution," in Ocean Acoustic Interference Phenomena and Signal Processing, edited by W. A. Kuperman and G. L. D'Spain (AIP, New York, 2002), Chap. 5, pp. 137-148.

${ }^{4}$ D. Rouseff and C. V. Leigh, "Using the waveguide invariant to analyze lofargrams," in Proceedings of the Oceans '02, Biloxi, MS (2002), pp. IV.2239-IV.2243.

${ }^{5}$ G. L. D'Spain and W. A. Kuperman, "Application of waveguide invariants to analysis of spectrograms from shallow water environments that vary in range and azimuth," J. Acoust. Soc. Am. 106, 2454-2468 (1999).

${ }^{6}$ T. C. Yang, "Beam intensity striations and applications," J. Acoust. Soc. Am. 113, 1342-1352 (2003).

${ }^{7}$ A. Thode, "Source ranging with minimal environmental information using a virtual receiver and waveguide invariant theory," J. Acoust. Soc. Am. 108, 1582-1594 (2000).

${ }^{8}$ L. M. Zurk and B. H. Tracey, "Depth-shifting of shallow water waveguide source observations," J. Acoust. Soc. Am. 118, 2224-2233 (2005).

${ }^{9}$ J. E. Quijano, L. M. Zurk, and D. Rouseff, "Demonstration of the invariance principle for active sonar," J. Acoust. Soc. Am. 123, 1329-1337 (2008).

${ }^{10} \mathrm{~J}$. Turgut and R. Gauss, "Investigation of bistatic invariance principles for active sonars," J. Acoust. Soc. Am. 125, 2704-2704 (2009).

${ }^{11}$ C. He, J. E. Quijano, and L. M. Zurk, "Enhanced Kalman filter algorithm using the invariance principle," IEEE J. Ocean. Eng. 34, 575-585 (2009).

${ }^{12}$ R. Goldhahn, G. Hickman, and J. L. Krolik, "Waveguide invariant reverberation mitigation for active sonar," in IEEE International Conference on Acoustics, Speech and Signal Processing, Honolulu, HI (2007), pp. II941-II-944.

${ }^{13}$ L. M. Zurk, D. Rouseff, J. E. Quijano, and G. Greenwood, "Bistatic invariance principle for active sonar geometries," in Proceedings of the 8th European Conference on Underwater Acoustics '06, Carvoeiro, Portugal (2006), pp. 787-791.

${ }^{14}$ J. S. Rogers and J. L. Krolik, "A study of active sonar reverberation using ultrasonic experiments in a shallow-water tank," in Proceedings of the Oceans '07, Scotland, UK (2007), pp. 1-5.

${ }^{15}$ K. D. LePage, P. Neumann and C. W. Holland, "Broad-band time domain modeling of sonar clutter in range dependent waveguides," in Proceedings of the Oceans '06, Boston, MA (2006), pp. 1-5.

${ }^{16}$ T. Oesterlein, C. He, J. E. Quijano, R. Campbell, Jr., L. M. Zurk, and M. Siderius, "Extraction of time-frequency target features," in Conference Record of the 39th Asilomar Conference on Signals, Systems and Computers '09, Pacific Grove, CA (2009).

${ }^{17}$ F. B. Jensen, W. A. Kuperman, M. B. Porter, and H. Schmidt, Computational Ocean Acoustics, 1st ed. (Springer-Verlag, New York, 2000), Chap. 5, pp. 257-281. 
Copyright of Journal of the Acoustical Society of America is the property of American Institute of Physics and its content may not be copied or emailed to multiple sites or posted to a listserv without the copyright holder's express written permission. However, users may print, download, or email articles for individual use. 